\title{
ON THE EIGENVALUES OF A MATRIX WITH PRESCRIBED SINGULAR VALUES ${ }^{1}$
}

\section{ALFRED HORN}

The singular values of a matrix $A$ (with complex elements) are the non-negative square roots of the eigenvalues of $A^{*} A$, where $A^{*}$ is the conjugate transpose of $A$. Let us call a pair of $n$-tuples $\left(\lambda_{1}, \cdots, \lambda_{n}\right)$, $\left(\alpha_{1}, \cdots, \alpha_{n}\right)$ allowable if there exists an $n$th order matrix with eigenvalues $\lambda_{1}, \cdots, \lambda_{n}$ and singular values $\alpha_{1}, \cdots, \alpha_{n}$. (Weyl [1] has proved that if $\left(\lambda_{1}, \cdots, \lambda_{n}\right),\left(\alpha_{1}, \cdots, \alpha_{n}\right)$ is allowable and if

$$
\alpha_{1} \geqq \cdots \geqq \alpha_{n} \geqq 0 \quad \text { and } \quad\left|\lambda_{1}\right| \geqq \cdots \geqq\left|\lambda_{n}\right| \text {, }
$$

then

$$
\begin{aligned}
& \left|\lambda_{1}\right| \cdots \cdot\left|\lambda_{k}\right| \leqq \alpha_{1} \cdots \cdot \alpha_{k} \quad \text { for } 1 \leqq k \leqq n, \\
& \left|\lambda_{1}\right| \cdots \cdot\left|\lambda_{n}\right|=\alpha_{1} \cdots \cdot \alpha_{n} .
\end{aligned}
$$

Dr. Seymour Sherman has raised the question as to whether these are the only general relations between an allowable pair. We are going to answer this question in the affirmative (Theorem 3). ${ }^{2}$ Theorem 4 shows that the above inequalities also characterize the sequence of principal minors of a positive definite Hermitian matrix with prescribed eigenvalues.

Two matrices $M, N$ are said to be unitarily equivalent (notation: $M \sim N)$ if there exist unitary matrices $U, V$ such that $M=U N V$. By the diagonal of a matrix $\left(A_{i, j}\right)$ we mean the $n$-tuple $\left(A_{1,1}, \cdots\right.$, $\left.A_{n, n}\right)$. A triangular matrix is one such that all elements below the diagonal vanish. It is easily proved that if $\left(x_{1}, \cdots, x_{n}\right)$ is a permutation of $\left(y_{1}, \cdots, y_{n}\right)$, then any triangular matrix with diagonal $\left(x_{1}, \cdots, x_{n}\right)$ is unitarily equivalent with a triangular matrix with diagonal $\left(y_{1}, \cdots, y_{n}\right)$.

Theorem 1. If the pair $\left(\lambda_{1}, \cdots, \lambda_{n}\right),\left(\alpha_{1}, \cdots, \alpha_{n}\right)$ satisfies (1), (2), (3) and if $\alpha_{n}>0$ and $\lambda_{i}>0$ for $1 \leqq i \leqq n$, then this pair is allowable.

Proof. ${ }^{3}$ Let $D$ be the diagonal matrix with diagonal $\left(\alpha_{1}, \cdots, \alpha_{n}\right)$. It is known that a matrix $A$ has singular values $\alpha_{1}, \cdots, \alpha_{n}$ if and

Presented to the Society, November 29, 1952; received by the editors October 21, 1952 and, in revised form, June 16, 1953.

1 This paper was written while the author received partial support from the Office of Naval Research.

2 Partial results in this direction were obtained by Parker [2]. For other proofs of Weyl's theorem see [3] and [4].

${ }^{3}$ This proof was kindly supplied by the referee. It uses the author's idea but is much simpler than the original argument. 
only if $A \sim D$ (for example, use the polar decomposition of $A$ ). Therefore we need only find a triangular matrix $A$ with diagonal $\left(\lambda_{1}, \cdots, \lambda_{n}\right)$ such that $A \sim D$. We proceed by induction. The theorem is obvious for $n=1$. For $n=2$, the theorem takes the form: if $\alpha_{1} \geqq \lambda_{1}$ $\geqq \lambda_{2} \geqq \alpha_{2}>0$ and $\lambda_{1} \lambda_{2}=\alpha_{1} \alpha_{2}$, then there exists a number $\mu$ such that

$$
\left(\begin{array}{ll}
\lambda_{1} & \mu \\
0 & \lambda_{2}
\end{array}\right)
$$

has singular values $\alpha_{1}, \alpha_{2}$. In fact $\mu=\left(\alpha_{1}^{2}+\alpha_{2}^{2}-\lambda_{1}^{2}-\lambda_{2}^{2}\right)^{1 / 2}$ is such a number. Let now $m \geqq 3$ and assume the theorem true for $1 \leqq n \leqq m-1$. Let $\left(\lambda_{1}, \cdots, \lambda_{m}\right),\left(\alpha_{1}, \cdots, \alpha_{m}\right)$ satisfy the hypothesis of our theorem with $n=m$. Let $\sigma_{1}=\alpha_{1}$ and $\sigma_{i}=\alpha_{1} \cdots \alpha_{i} / \lambda_{2} \cdots \cdots \lambda_{i}$ for $2 \leqq i \leqq m-1$. Put $\sigma=\min \sigma_{i}, 1 \leqq i \leqq m-1$, and $\rho=\lambda_{1} \lambda_{m} / \sigma$. Suppose $\sigma$ is attained for $i=k$. It is easy to see by (1), (2), (3) that $\alpha_{1} \geqq \sigma \geqq \lambda_{1}$ $\geqq \lambda_{m} \geqq \rho \geqq \alpha_{m}>0$. Therefore

$$
\left(\begin{array}{ll}
\lambda_{1} & \mu \\
0 & \lambda_{m}
\end{array}\right) \sim\left(\begin{array}{ll}
\sigma & 0 \\
0 & \rho
\end{array}\right)
$$

for some $\mu$. Further it is easily seen that the pairs $\left(\sigma, \lambda_{2}, \cdots, \lambda_{k}\right)$, $\left(\alpha_{1}, \alpha_{2}, \cdots, \alpha_{k}\right)$ and $\left(\lambda_{k+1}, \cdots, \lambda_{m-1}, \rho\right),\left(\alpha_{k+1}, \cdots, \alpha_{m-1}, \alpha_{m}\right)$ satisfy the hypothesis of our theorem with $n=k, n=m-k$ respectively. Consequently, by the induction hypothesis,

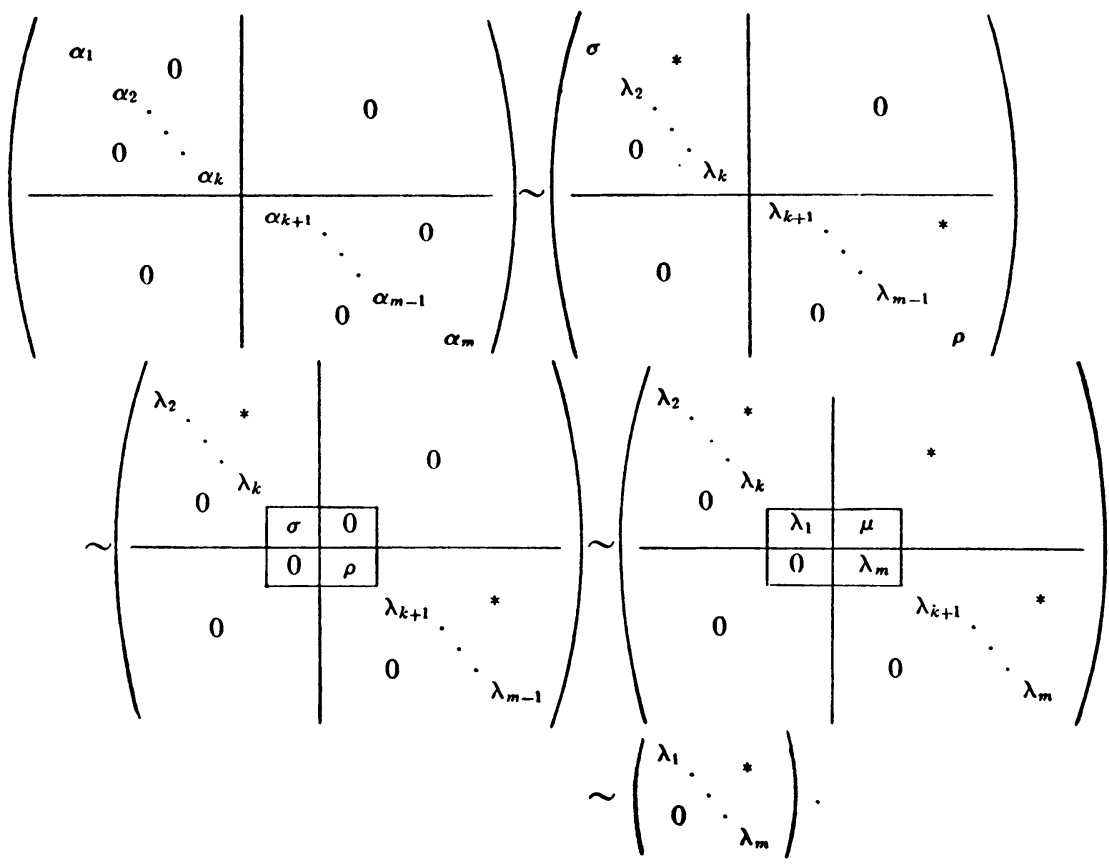


Theorem 2. If the pair $\left(\lambda_{1}, \cdots, \lambda_{n}\right),\left(\alpha_{1}, \cdots, \alpha_{n}\right)$ satisfies (1), (2), (3) and if $\lambda_{i} \geqq 0$, for $1 \leqq i \leqq n$, then this pair is allowable.

Proof. If $\alpha_{n}>0$, we need only cite Theorem 1 . Suppose $\alpha_{k}>0$ but $\alpha_{k+1}=\cdots=\alpha_{n}=0$. By (3), $\lambda_{n}=0$. Similarly by successively using (2), we find $\lambda_{k+1}=\cdots=\lambda_{n}=0$. Now suppose $\lambda_{m} \neq 0$ but $\lambda_{m+1}=0$, for some $m \leqq k$. Let $\beta=\left(\lambda_{1} \cdots \cdots \lambda_{m}\right) /\left(\alpha_{1} \cdots \cdots \alpha_{m-1}\right)$. Note that $\beta \leqq \alpha_{m}$. By Theorem 1 there exists an $m$ th order matrix $A$ with eigenvalues $\lambda_{1}, \cdots, \lambda_{m}$ and singular values $\alpha_{1}, \cdots, \alpha_{m-1}, \beta$. Let $U$ be a unitary matrix such that $U^{*} A^{*} A U$ is a diagonal matrix with diagonal elements $\alpha_{1}^{2}, \cdots, \alpha_{m-1}^{2}, \beta^{2}$. Let $B=U^{*} A U$. Then $B$ also has eigenvalues $\lambda_{1}, \cdots, \lambda_{m}$ and $B^{*} B=U^{*} A^{*} A U$. Now let $C$ be the $n$th order matrix with elements defined by: $C_{i, j}=B_{i, j}$ whenever $1 \leqq i \leqq m$, $1 \leqq j \leqq m, C_{m, m+1}=\gamma, C_{i, i+1}=\alpha_{i}$ whenever $m+1 \leqq i \leqq k$, and $C_{i, j}=0$ otherwise. Then $C$ has the eigenvalues $\lambda_{1}, \cdots, \lambda_{m}, 0, \cdots, 0$ while $C^{*} C$ is a diagonal matrix with diagonal elements $\alpha_{1}^{2}, \cdots, \alpha_{m-1}^{2}$, $\beta^{2}+\gamma^{2}, \alpha_{m+1}^{2}, \cdots, \alpha_{k}^{2}, 0, \cdots, 0$. If we choose $\gamma$ so that $\beta^{2}+\gamma^{2}=\alpha_{m}^{2}$, then $C$ has all the desired properties. ${ }^{4}$

Theorem 3. If the pair $\left(\lambda_{1}, \cdots, \lambda_{n}\right),\left(\alpha_{1}, \cdots, \alpha_{n}\right)$ satisfy (1), (2), (3), then this pair is allowable.

Proof. By Theorem 2, there exists a matrix $A$ with eigenvalues $\left|\lambda_{1}\right|, \cdots,\left|\lambda_{n}\right|$ and singular values $\alpha_{1}, \cdots, \alpha_{n}$. We may assume $A$ is triangular. Let $V$ be the diagonal matrix whose $i$ th diagonal element is $\lambda_{i} /\left|\lambda_{i}\right|$ if $\lambda_{i} \neq 0$, or 1 if $\lambda_{i}=0$. Then $V A$ obviously has eigenvalues $\lambda_{1}, \cdots, \lambda_{n}$ and singular values $\alpha_{1}, \cdots, \alpha_{n}$.

THEOREM 4. Suppose $x_{1} \geqq \cdots \geqq x_{n}>0$ and $y_{i}>0$ for $1 \leqq i \leqq n$. Then the following statements are equivalent:

(a) There exists an nth order matrix with eigenvalues $y_{1}, \cdots, y_{n}$ and singular values $x_{1}, \cdots, x_{n}$,

(b) $y_{i_{1}} \cdot \cdots \cdot y_{i_{k}} \leqq x_{1} \cdot \cdots \cdot x_{k}$ whenever $1 \leqq i_{1}<\cdots<i_{k} \leqq n, 1 \leqq k$ $\leqq n$, and

$$
y_{1} \cdot \cdots \cdot y_{n}=x_{1} \cdot \cdots \cdot x_{n} .
$$

(c) There exists a Hermitian matrix $A=\left(A_{i, j}\right)$ with eigenvalues $x_{1}, \cdots, x_{n}$ such that

$$
y_{1} \cdots y_{k}=\left|\begin{array}{ccc}
A_{1,1} \cdots & A_{1, k} \\
\cdot & \cdot & \cdot \\
A_{k, 1} \cdots & A_{k, k}
\end{array}\right| \quad \text { for } 1 \leqq k \leqq n .
$$

4 This proof was suggested by Professor I. Kaplansky. 
Proof. Weyl's theorem and Theorem 1 shows the equivalence of (a) and (b). Consequently if (a) holds, there exists a triangular matrix $B$ with eigenvalues $\left(y_{1}^{1 / 2}, \cdots, y_{n}^{1 / 2}\right)$ and singular values $\left(x_{1}^{1 / 2}, \cdots, x_{n}^{1 / 2}\right)$. By a well known theorem on determinants, the matrix $A=B^{*} B$ satisfies the conditions of (c). Conversely if $A$ satisfies the conditions of (c), we have $A=B^{*} B$, where $B$ is a triangular matrix [5, p. 20]. The diagonal of $B$ must be $\left(y_{1}^{1 / 2}, \cdots, y_{n}^{1 / 2}\right)$. Therefore $\left(y_{1}^{1 / 2}, \cdots, y_{n}^{1 / 2}\right)$, $\left(x_{1}^{1 / 2}, \cdots, x_{n}^{1 / 2}\right)$ is allowable. Another application of the equivalence between (a) and (b) completes the proof.

\section{REFERENCES}

1. H. Weyl, Inequalities between the two kinds of eigenvalues of a linear transformation, Proc. Nat. Acad. Sci. U.S.A. vol. 35 (1949) pp. 408-411.

2. W. V. Parker, The characteristic roots of matrices, Duke Math. J. vol. 12 (1945) pp. 509-526.

3. A. Horn, On the singular values of a product of completely continuous operators, Proc. Nat. Acad. Sci. U.S.A. vol. 36 (1950) pp. 374-375.

4. C. Visser and A. C. Zaanen, On the eigenvalues of compact linear transformations, Nederl. Akad. Wetensch. vol. 14 (1952) pp. 71-78.

5. F. D. Murnaghan, The theory of group representations, Baltimore, 1938.

The Institute for Advanced StUdy 